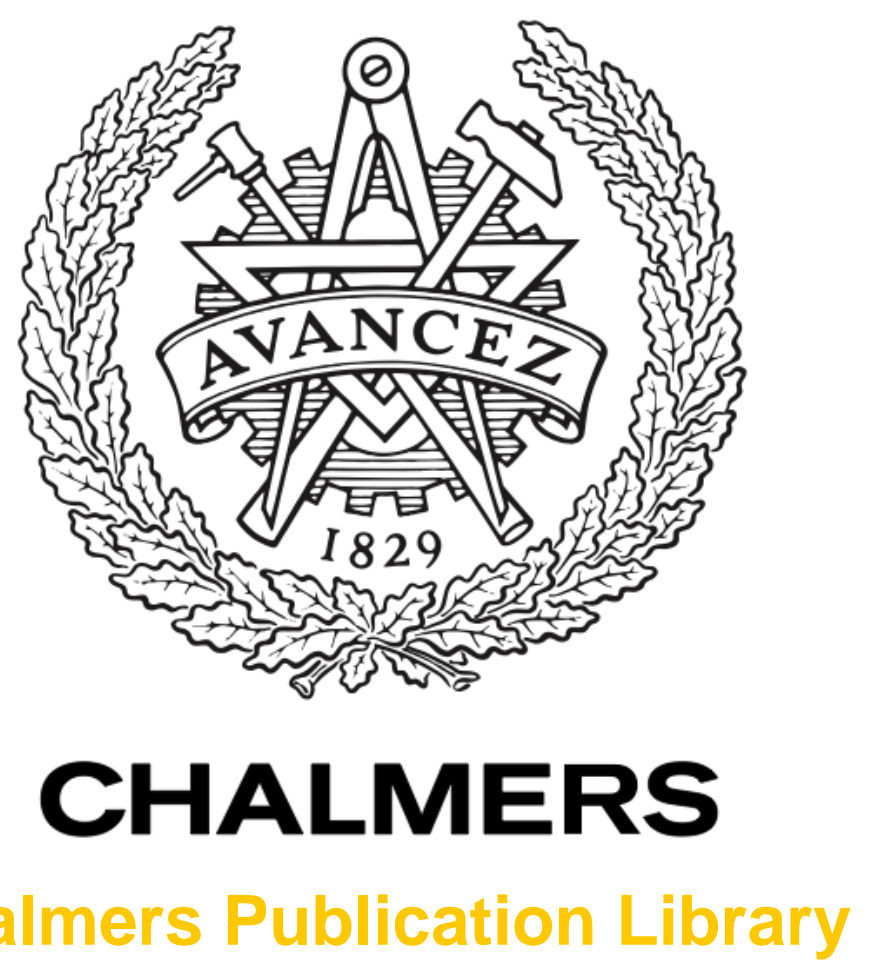

\title{
Continuous wave-pumped wavelength conversion in low-loss silicon nitride waveguides
}

This document has been downloaded from Chalmers Publication Library (CPL). It is the author's version of a work that was accepted for publication in:

\section{Optics Letters (ISSN: 0146-9592)}

Citation for the published paper:

Krückel, C. ; Torres Company, V. ; Andrekson, P. et al. (2015) "Continuous wave-pumped wavelength conversion in low-loss silicon nitride waveguides". Optics Letters, vol. 40(6), pp. $875-878$.

Downloaded from: http://publications.lib.chalmers.se/publication/214793

Notice: Changes introduced as a result of publishing processes such as copy-editing and formatting may not be reflected in this document. For a definitive version of this work, please refer to the published source. Please note that access to the published version might require a subscription. 


\title{
Continuous wave-pumped wavelength conversion in low-loss silicon nitride waveguides
}

\author{
Clemens J. Krückel, ${ }^{1, *}$ Víctor Torres-Company, ${ }^{1}$ Peter A. Andrekson, ${ }^{1}$ Daryl T. Spencer, ${ }^{2}$ Jared F. Bauters, ${ }^{2}$ \\ Martijn J. R. Heck, ${ }^{2}$ and John E. Bowers ${ }^{2}$ \\ ${ }^{1}$ Photonics Laboratory, Department of Microtechnology and Nanoscience, Chalmers University of Technology, SE-412 96 Gothenburg, Sweden \\ ${ }^{2}$ Department of Electrical and Computer Engineering, University of California, Santa Barbara, California 93106, USA \\ ${ }^{*}$ Corresponding author: kruckel@chalmers.se
}

Received December 16, 2014; revised January 26, 2015; accepted January 28, 2015;

posted January 28, 2015 (Doc. ID 230655); published March 3, 2015

\begin{abstract}
In this Letter we introduce a complementary metal-oxide semiconductor (CMOS)-compatible low-loss $\mathrm{Si}_{3} \mathrm{~N}_{4}$ waveguide platform for nonlinear integrated optics. The waveguide has a moderate nonlinear coefficient of $285 \mathrm{~W} / \mathrm{km}$, but the achieved propagation loss of only $0.06 \mathrm{~dB} / \mathrm{cm}$ and the ability to handle high optical power facilitate an optimal waveguide length for wavelength conversion. We observe a constant quadratic dependence of the four-wave mixing (FWM) process on the continuous-wave (CW) pump when operating in the C-band, which indicates that the waveguide has negligible high-power constraints owing to nonlinear losses. We achieve a conversion efficiency of $-26.1 \mathrm{~dB}$ and idler power generation of $-19.6 \mathrm{dBm}$. With these characteristics, we present for the first time, to the best of our knowledge, CW-pumped data conversion in a non-resonant $\mathrm{Si}_{3} \mathrm{~N}_{4}$ waveguide. (C) 2015 Optical Society of America

OCIS codes: (130.3120) Integrated optics devices; (190.4360) Nonlinear optics, devices; (190.4380) Nonlinear optics, four-wave mixing; (190.4390) Nonlinear optics, integrated optics.

http://dx.doi.org/10.1364/OL.40.000875
\end{abstract}

Photonic integration can lead to drastic reductions in power consumption and the size of optical systems. The integration of photonic systems showing nonlinear phenomena such as four-wave mixing (FWM) enables useful applications for all-optical signal processing such as wavelength conversion [1] or signal regeneration [2] at the chip level. Material platforms suitable for nonlinear integrated optics, including silicon-based compounds

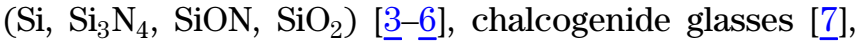
and III-V semiconductors (AlGaAs) []], have been extensively studied for decades. In addition, some of the above materials could be hybridized with extra layers, such as organic composites [9] , to further improve the nonlinear performance.

Using the mature technologies of the complementary metal-oxide semiconductor (CMOS) processing platform for integrated optics enables the option for mass production and thus tremendously reduces processing costs. To better understand why silicon nitride is our chosen material, let us turn our attention to the nonlinear phase shift $\Phi_{\mathrm{NL}}=\gamma P L_{\text {eff }}$. This product of nonlinear coefficient $\gamma$, power level $P$, and effective length $L_{\text {eff }}$ must be maximized to achieve high FWM efficiency. As the nonlinear Kerr coefficient of $\mathrm{Si}_{3} \mathrm{~N}_{4}$ is one order of magnitude greater than the one in $\mathrm{SiO}_{2}$ [10], a higher $\gamma$ and a more efficient Kerr process is achieved in $\mathrm{Si}_{3} \mathrm{~N}_{4}$. Although $\mathrm{Si}$ has even higher nonlinearities, the nonlinear losses originating from two-photon absorption (TPA) and free carrier absorption at telecom wavelengths limit the maximum power levels $P$ that can be sent in this material. The need to operate at moderate power levels means that the energy in the wavelength-converted signal will not be high, regardless of the conversion efficiency (CE). It is of course possible to remove the photogenerated carriers in silicon waveguides by applying an electric field across the structure [11], but this requires additional manufacturing steps. These limitations do not exist in silicon nitride owing to its larger bandgap, providing a transparency window that extends to the ultraviolet [12]. In addition, the high index contrast to $\mathrm{SiO}_{2}$ allows for high optical confinement with the option to tailor waveguide dispersion [13].

Indeed, wavelength conversion by $\mathrm{FWM}$ in $\mathrm{Si}_{3} \mathrm{~N}_{4}$ waveguides has been presented previously. But in order to achieve high CE, a pulsed pump with high peak power has been used in straight waveguides [14]. Alternatively, cavity enhancement of a continuous-wave pump has been shown in resonating structures [15]. Comb generation by cascaded FWM has been demonstrated in [15-17]. These examples clearly indicate the potential of this material for nonlinear integrated optics. However, CW wavelength conversion in non-resonant silicon nitride waveguide structures has not been investigated thoroughly. In this Letter we present CW-pumped wavelength conversion using the waveguides previously developed in [18]. This work extends the research presented in [19] by providing additional measurements and a more detailed comparison with numerical simulations. These original waveguide designs featured low propagation loss [18] so that a large effective length $L_{\text {eff }}$ of up to a few meters could be achieved in a compact platform. Larger interaction length results in an increased nonlinear phase shift and thus a higher FWM efficiency. We critically test the performance of these devices in a data conversion experiment that shows for the first time, to the best of our knowledge, CW-pumped wavelength conversion in a non-resonating $\mathrm{Si}_{3} \mathrm{~N}_{4}$ waveguide.

The low propagation loss of this platform is achieved by using very thin waveguide dimensions. In this Letter we use a $\mathrm{Si}_{3} \mathrm{~N}_{4}$ waveguide core cross section with a height of $100 \mathrm{~nm}$ and a width of $2.8 \mu \mathrm{m}$. The propagation loss is only $0.06 \mathrm{~dB} / \mathrm{cm}$. The waveguide cross section of the $\mathrm{Si}_{3} \mathrm{~N}_{4}$ core and $\mathrm{SiO}_{2}$ cladding is shown in Fig. 1(a). Waveguides with similar dimensions have been used previously for linear applications; design and fabrication were first presented in [18]. The weak field confinement 


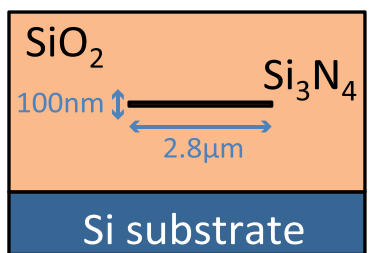

(a)

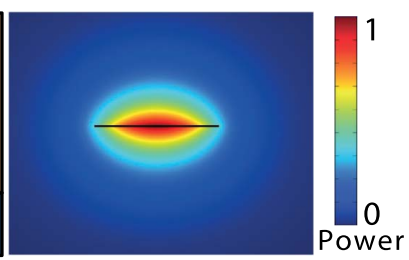

(b)

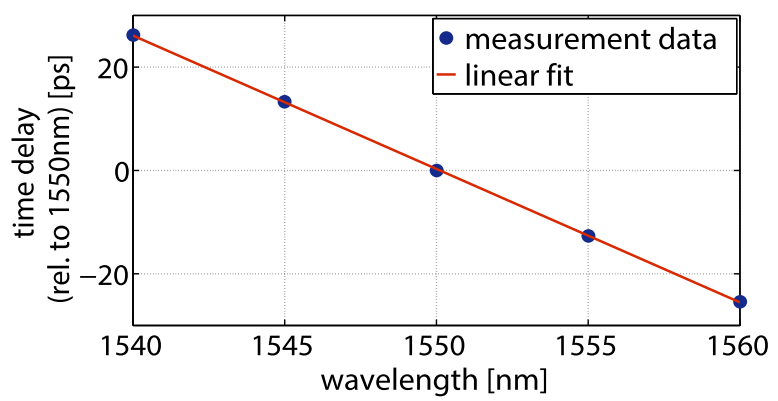

(c)
Fig. 1. (a) Schematic of waveguide cross section. (b) Simulation of power distribution of the fundamental TE-mode. (c) Measurement of time delay relative to $1550 \mathrm{~nm}$ versus wavelength.

in our design (only around $12 \%$ of the optical power is guided in the $\mathrm{Si}_{3} \mathrm{~N}_{4}$ core) leads to low scattering interaction of the optical field with the thin waveguide sidewalls. The waveguide has single mode properties; simulations of the power distribution of the fundamental TE-mode are shown in Fig. 1(b). Since most of the power is in the cladding, we investigated the impact of having a change in its refractive index. A $5 \%$ variation of the cladding refractive index modifies the power confinement by less than $1.5 \%$.

In the experiments, light is launched into the waveguide over tapered fibers with a coupling loss of $2.8 \mathrm{~dB}$ per facet. The propagation and coupling losses were evaluated with the cutback method. Waveguides of several meter lengths were coiled in spiral form with a minimum bending radius of $1 \mathrm{~mm}$ on a chip with a reduced footprint of $1.5 \mathrm{~cm} \times 1.5 \mathrm{~cm}$. As the waveguide has a polarization extinction ratio of more than $40 \mathrm{~dB}$, we optimized the launched light for the lower-loss TE-polarization in all experiments. It is worth mentioning that even lower losses were achieved in the next generation of this platform, with thinner core thicknesses, with wafer bonding of high-quality thermal $\mathrm{SiO}_{2}$, and at longer wavelengths, where the hydrogen bond absorption is lower [20], but the performance has not been tested for nonlinear optics.

The dispersion properties of this design were studied experimentally in a $6 \mathrm{~m}$ long waveguide by measuring the time delay versus wavelength using the time-of-flight technique. The results are presented in Fig. 1(c). From the measurement data we extracted a normal group velocity dispersion (GVD) of $-0.4 \mathrm{ps} / \mathrm{nm} / \mathrm{m}$ at $1550 \mathrm{~nm}$ and a dispersion slope of $1.4 \mathrm{ps} / \mathrm{nm}^{2} / \mathrm{m}$. The GVD is in fair agreement with the expected value of $-0.56 \mathrm{ps} /$ $\mathrm{nm} / \mathrm{m}$ computed from mode solver simulations. We measured a nonlinear coefficient $\gamma$ of $285 \mathrm{~W} / \mathrm{km}$ for this low-loss $\mathrm{Si}_{3} \mathrm{~N}_{4}$ waveguide in a dual pump experiment [21]. Compared to other techniques, the dual pump experiment amplifies the two pumps independently.
Nonlinear effects occurring in the amplifiers become irrelevant. The results are in agreement with [10], where nonlinearities of different core thicknesses were investigated. To determine the optimal waveguide length for the following nonlinear experiments, we simulated the FWM-based idler generation by solving the nonlinear Schrödinger equation (NLSE) with the split-step Fourier method. As waveguide parameters for the simulations, we used the measured values of losses, dispersion, and nonlinear coefficient stated above. The simulated idler power as a function of waveguide length (Fig. 2) indicates that there is an optimum waveguide length of around $0.8 \mathrm{~m}$. Above this length, a clear impact of the linear loss on the nonlinear FWM process is visible. For the theoretical model we used $33.3 \mathrm{dBm}$ for the pump $(1563 \mathrm{~nm})$ and $18.9 \mathrm{dBm}$ for the signal $(1562 \mathrm{~nm})$, which correspond to the power values used in the measurements. Next, we assessed the $\mathrm{CE}$ of the waveguides in an FWM experiment by launching a signal-pump pair into the waveguide in a degenerate pump configuration. A waveguide of $1 \mathrm{~m}$ length was used in this nonlinear measurement. To minimize the impact of dispersion, a detuning of $1 \mathrm{~nm}$ between the waves was chosen. Signal and pump were amplified independently and combined in a wavelength-division multiplexing coupler before launching into the waveguide. While keeping the signal at a constant $18.9 \mathrm{dBm}$, the pump power was varied. At the output of the chip, we analyzed the CE, optimistically defined as $P_{\text {idler }}^{\text {out }} / P_{\text {signal }}^{\text {out }}$, and the absolute idler power as a function of launched pump power. As expected, the maximum $\mathrm{CE}$ was $-26.1 \mathrm{~dB}$, corresponding to a launched pump power of $33.3 \mathrm{dBm}$ as shown in Fig. 3(a). This results in a converted idler power of $-19.6 \overline{\mathrm{dBm}}$ for the launched signal power of $18.9 \mathrm{dBm}$ [Fig. 3(a)]. In the figure, measurement data are compared with the theoretical model, which shows a constant quadratic dependence on the pump power even up to $33.3 \mathrm{dBm}$. This indicates that the saturation effects owing to TPA or carrier effects are negligible. These CE results offer incomplete information about the absolute generated idler power. In spite of a relatively low $\mathrm{CE}$, the advantage of our platform is that it can handle high $\mathrm{CW}$ power, thus allowing us to achieve high idler powers. The results presented here provide a $5.5 \mathrm{~dB}$ improvement with respect to the work in [19], where a $2 \mathrm{~m}$ long waveguide was used instead. This is in agreement with the simulations shown in Fig. 2.

An important aspect of any FWM-based wavelength converter is the impact of chromatic dispersion. We assess this by changing the wavelength spacing of the

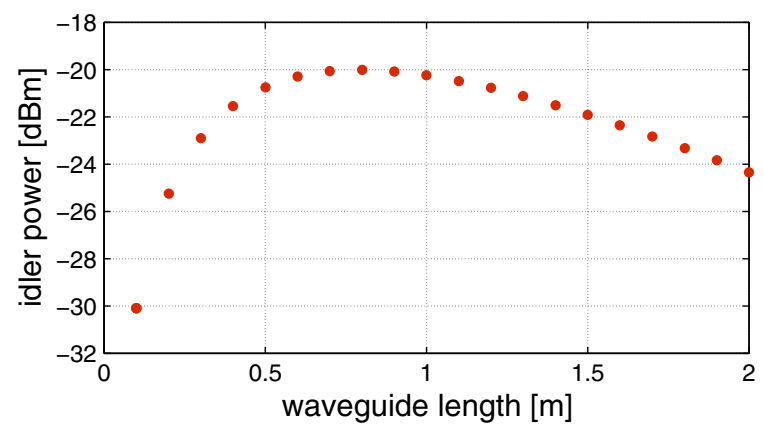

Fig. 2. Numerical simulation of maximum generated idler power versus length of waveguide. 


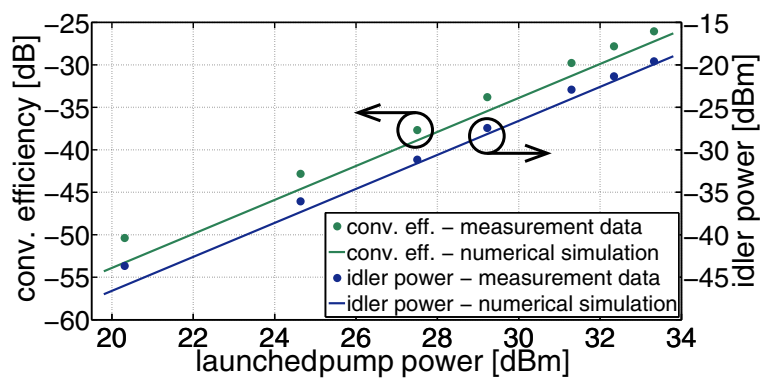

(a)

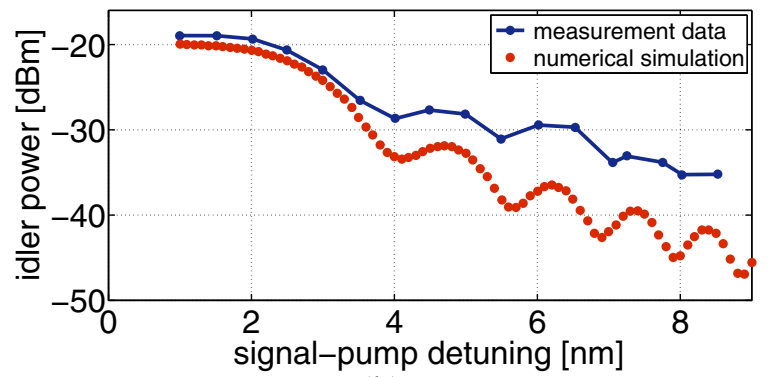

(b)

Fig. 3. (a) Measurement and numerical simulation of output conversion efficiency and idler power versus launched pump power in a $1 \mathrm{~m}$ long waveguide. (b) Measurement and numerical simulation of idler power versus wavelength separation of signal and pump.

signal-pump pair. Detuning signal and pump results in an additional phase mismatch that reduces the FWM CE. The launched power levels of signal and pump were kept constant at 18.9 and $33.3 \mathrm{dBm}$. The signal wavelength was decreased from $1562 \mathrm{~nm}$ while keeping the pump wavelength at $1563 \mathrm{~nm}$. In Fig. 3(b) the results of the measured converted idler power versus signal-pump detuning are plotted. The $3 \mathrm{~dB}$-bandwidth of the idler power is reached at a signal-pump spacing of $2.7 \mathrm{~nm}$. We compared the measurements with the theoretical model of the system as shown in Fig. 3(b). Each point in the simulation curve is an independent solution of the NLSE for a different wavelength separation of signal and pump. The value of the GVD in the simulations is tuned to match the locations of the minimums in the measurement ripples.

Finally, to assess the performance of the waveguide in a more practical scenario, we carried out a wavelength data conversion experiment. The setup for this measurement is shown in Fig. 4(a). The signal wave is modulated with $10 \mathrm{~Gb} / \mathrm{s}$ non-return-to-zero (NRZ) on-off keying (OOK) data, amplified, and then launched together with the amplified pump wave into a $2 \mathrm{~m}$ long waveguide. After the chip, the idler is filtered out from the spectrum with an optical bandpass filter (OBF) and launched into the receiver stage at different power levels, controlled with a variable optical attenuator (VOA). We use a preamplifier-based receiver stage in which the idler is optically amplified and filtered, and then amplified after the photo detector in the electrical domain. To evaluate the bit-error rate (BER) of the data conversion, we compared the data at the idler wavelength with the original generated bit sequence in front of the chip. Figure 4(b) shows the BER as a function of launched power into the pre-amplified receiver. Error-free (BER of $10^{-9}$ ) data

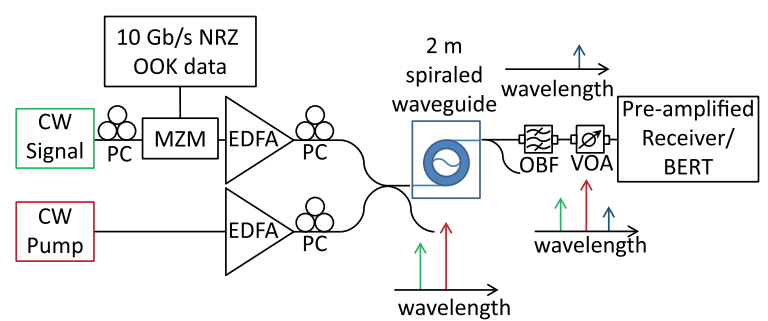

(a)

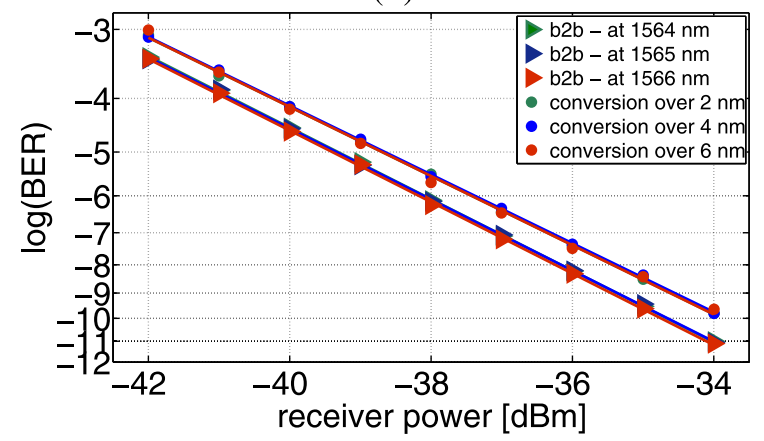

(b)

Fig. 4. (a) Schematic of experimental setup for BER measurements of data wavelength conversion $(\mathrm{PC}=$ polarization controller; $\mathrm{MZM}=$ Mach-Zehnder electro-optic modulator; EDFA = erbium-doped fiber amplifier; OBP = optical bandpass filter; $\mathrm{VOA}=$ variable optical attenuator; BERT $=$ bit error rate tester). (b) BER curves for back-to-back (b2b) transmission and wavelength conversion over 2,4 , and $6 \mathrm{~nm}$ spacing.

conversion is achieved at a receiver power of $-34.6 \mathrm{dBm}$ for up to $6 \mathrm{~nm}$ conversion width. A receiver penalty of $0.8 \mathrm{~dB}$ of converted data with respect to back-to-back (b2b) transmission can be seen. In comparison to data conversion in other platforms such as $\mathrm{Si}$, where dispersionengineered waveguides have been used [22], in our experiments the high normal dispersion significantly limits our conversion bandwidth. However, the waveguide dimensions in our design have not been optimized for high bandwidth wavelength conversion.

In summary, we have shown that the CMOS-compatible low-loss $\mathrm{Si}_{3} \mathrm{~N}_{4}$ waveguide presented in [18], originally developed for linear optics applications, is also well suited for nonlinear optics applications (particularly FWM-based wavelength conversion). Although the nonlinear coefficient $(285 \mathrm{~W} / \mathrm{km})$ is small compared to other integrated nonlinear platforms, we achieved an output $\mathrm{CE}$ of $-26.1 \mathrm{~dB}$ and an absolute idler power of $-19.6 \mathrm{dBm}$. Our experiments illustrate the relevance of having a long effective length and high power-handling capabilities. With these characteristics we present, for the first time, $\mathrm{CW}$ data conversion in a non-resonant $\mathrm{Si}_{3} \mathrm{~N}_{4}$ waveguide.

This work is supported by the European Research Council under grant agreement ERC-2011-AdG-291618 PSOPA and the Wallenberg Foundation. Víctor TorresCompany gratefully acknowledges funding from the Swedish Research Council (VR).

\section{References}

1. H. Fukuda, K. Yamada, T. Shoji, M. Takahashi, T. Tsuchizawa, T. Watanabe, J. Takahashi, and S. Itabashi, Opt. Express 13, 4629 (2005). 
2. R. Salem, M. A. Foster, A. C. Turner, D. F. Geraghty, M. Lipson, and A. L. Gaeta, Nat. Photonics 2, 35 (2007).

3. J. Leuthold, C. Koos, and W. Freude, Nat. Photonics 4, 535 (2010).

4. Y. Shoji, T. Ogasawara, T. Kamei, Y. Sakakibara, S. Suda, K. Kintaka, H. Kawashima, M. Okano, T. Hasama, H. Ishikawa, and M. Mori, Opt. Express 18, 5668 (2010).

5. D. J. Moss, R. Morandotti, A. L. Gaeta, and M. Lipson, Nat. Photonics 7, 597 (2013).

6. D. Y. Oh, D. Sell, H. Lee, K. Y. Yang, S. A. Diddams, and K. J. Vahala, Opt. Lett. 39, 1046 (2014).

7. B. Eggleton, B. Luther-Davies, and K. Richardson, Nat. Photonics 5, 141 (2011).

8. P. Apiratikul, J. J. Wathen, G. A. Porkolab, B. Wang, L. He, T. E. Murphy, and C. J. K. Richardson, Opt. Express 22, 26814 (2014).

9. C. Koos, P. Vorreau, T. Vallaitis, P. Dumon, W. Bogaerts, R. Baets, B. Esembeson, I. Biaggio, T. Michinobu, F. Diederich, W. Freude, and J. Leuthold, Nat. Photonics 3, 216 (2009).

10. M. Tien, J. F. Bauters, M. J. R. Heck, D. J. Blumenthal, and J. E. Bowers, Opt. Express 18, 23562 (2010).

11. A. Gajda, L. Zimmermann, M. Jazayerifar, G. Winzer, H. Tian, R. Elschner, T. Richter, C. Schubert, B. Tillack, and K. Petermann, Opt. Express 20, 13100 (2012).

12. R. Soref, Nat. Photonics 4, 495 (2010).

13. Y. Okawachi, K. Saha, J. S. Levy, Y. H. Wen, M. Lipson, and A. L. Gaeta, Opt. Lett. 36, 3398 (2011).

14. J. S. Levy, A. Gondarenko, A. C. Turner-Foster, M. A. Foster, A. L. Gaeta, and M. Lipson, in Proceedings of the Conference on Lasers and Electro-Optics/International Quantum Electronics Conference, Baltimore, MD (Optical Society of America, 2009).

15. J. S. Levy, A. Gondarenko, M. A. Foster, A. C. Turner-Foster, A. L. Gaeta, and M. Lipson, Nat. Photonics 4, 37 (2009).

16. F. Ferdous, H. Miao, D. E. Leaird, K. Srinivasan, J. Wang, L. Chen, L. T. Varghese, and A. M. Weiner, Nat. Photonics 5, 770 (2011).

17. J. Pfeifle, V. Brasch, M. Lauermann, Y. Yu, D. Wegner, T. Herr, K. Hartinger, P. Schindler, J. Li, D. Hillerkuss, R. Schmogrow, C. Weimann, R. Holzwarth, W. Freude, J. Leuthold, T. J. Kippenberg, and C. Koos, Nat. Photonics 8, 375 (2014).

18. J. F. Bauters, M. J. R. Heck, D. John, M. Tien, A. Leinse, R. Heideman, D. Blumenthal, and J. E. Bowers, in Proceedings of European Conference on Optical Communication, Turin, Italy (ECOC, 2010), paper We.8.E.6.

19. C. Krückel, V. Torres-Company, P. Andrekson, J. Bovington, J. Bauters, M. Heck, and J. Bowers, in Proceedings of the Conference on Lasers and Electro-Optics, San Jose, CA (Optical Society of America, 2014).

20. J. Bauters, M. J. R. Heck, D. D. John, J. S. Barton, C. M. Bruinink, A. Leinse, R. G. Heideman, D. J. Blumenthal, and J. E. Bowers, Opt. Express 19, 24090 (2011).

21. A. Boskovic, S. V. Chernikov, J. R. Taylor, L. GrunerNielsen, and O. A. Levring, Opt. Lett. 21, 1966 (1996).

22. N. Ophir, J. Chan, K. Padmaraju, A. Biberman, A. C. Foster, M. A. Foster, M. Lipson, A. L. Gaeta, and K. Bergman, IEEE Photon. Technol. Lett. 23, 73 (2011). 